\title{
Social Balance and the Bernoulli Equation
}

\section{J. J. P. Veerman}

\begin{abstract}
Since the 1940s there has been an interest in the question of why social networks often give rise to two antagonistic factions. Recently a dynamical model of how and why such a balance might occur was developed. This note provides an introduction to the notion of social balance and a new (and simplified) analysis of that model. This new analysis allows us to choose general initial conditions, as opposed to the symmetric ones previously considered. We show that for general initial conditions, four factions will evolve instead of two. We characterize the four factions, and give an idea of their relative sizes.
\end{abstract}

1. INTRODUCTION. Since the 1940s there has been an interest in the question of why finite social networks often give rise to two antagonistic factions [3, 5, 6]. Motivated by insights from the field of social psychology (most notably by Heider [6]), Harary [5] and Cartwright and Harary [3] developed a formal graph-theoretical framework for social balance that was consistent with Heider's ideas. They were able to prove that a sign symmetric network is balanced if and only if it has (at most) two factions (see below for the definitions). This became know as the structure theorem. Until recently however, a dynamical model of how and why such a balance occurs was lacking.

The field received an impetus in 2011 when in [8], Marvel et al. successfully analyzed the dynamics of the matrix differential equation

$$
\dot{X}=X^{2} ; \quad X(0)=X_{0} .
$$

The interpretation of this model is that the entries of the $n \times n$ matrix $X$ represent the opinions of individuals $\{1, \ldots, n\}$ towards one another. The value of the entry $x_{i j}$ of $X$ indicates the strength of the friendliness $[\mathbf{8}]$ of individual $i$ towards individual $j$. The modeling becomes clear if we write out the differential equation for one entry:

$$
\dot{x}_{i j}=\sum_{k=1}^{n} x_{i k} x_{k j} .
$$

Thus individual $i$ communicates somehow with all individuals $k \in\{1, \ldots, n\}$. If $i$ 's feeling about $k$ is positive, then $k$ 's feeling about $j$ will pull $i$ 's opinion in the same direction. On the other hand, if $i$ 's opinion about $k$ is negative, then $i$ 's opinion about $j$ will change in the direction opposite to $k$ 's opinion about $j$. This "implies roughly that ... one's friends' friends will tend to become one's friends and one's enemies' enemies also one's friends, and one's enemies' friends and one's friends' enemies will tend to become one's enemies" [10]. Since friendliness is difficult to quantify, one is led to study the problem with the initial value $X_{0}$ being a random matrix.

To perform the analysis of equation (1), the authors of [8] assumed the matrix $X_{0}$ is symmetric. (This was later extended to normal matrices [14].) The analysis itself now proceeded in two parts. The first is to solve the differential equation (1D) by considering it as a special case of a matrix Riccati equation (see [11]). This solution is subject to certain conditions, the most important of which are that $X_{0}$ has a positive real eigenvalue, and that the largest of these real eigenvalues, $\lambda$, has algebraic and geometric 
multiplicity 1 . The second part of the analysis is to show that for a random symmetric matrix $X_{0}$ these hypotheses hold with probability tending to 1 as the dimension $n$ grows unbounded. This involves relying on fairly subtle arguments about eigenvalues of random symmetric matrices (see [1]).

In this article we propose a simpler and more precise treatment of this problem. In Section 2 we give a very simple proof of a generalization of the structure theorem. In it the hypothesis that the network be sign symmetric has been dropped. In Section 3 we treat equation (1) not as a Riccati equation but as a special case of a Bernoulli equation (first mentioned in [2], see also [13] and other contemporary textbooks). This simplifies our treatment and has the advantage that we can characterize much more precisely than previously what happens for nonsymmetric (or nonnormal) initial conditions $X_{0}$. We make use of a recent study ([12]) of random (nonsymmetric) matrices to assert that also in this case with probability tending to 1 as the dimension $n$ tends to infinity, $X_{0}$ has a leading real, simple, positive eigenvalue. As a result, we are able to show that with overwhelming probability a random initial condition $X_{0}$ (not symmetric or normal) will lead to four factions. Two of these factions are similar to the factions in the symmetric case: they are cohesive in the sense that members of the same faction like each other. The other two factions are not: members of the same faction dislike each other. Finally, in Section 4, we show that if the two cohesive factions join forces, they can be expected to have a narrow majority. This last result is new.

Finally we remark on a later development. In [14] a slightly different model for social balance is proposed, namely the differential equation in (1) is replaced by $\dot{X}=$ $X X^{T}$. This model gives rise to two factions with high probability under general initial conditions. The analysis is substantially more complicated. It appears that the simplification we propose here does not help in the study of this model.

2. WHAT IS SOCIAL BALANCE? The signed directed graph $G$ on $n$ vertices is a collection $V$ of $n$ vertices together with a set $E$ of directed edges between certain ordered pairs of vertices whose weights are positive or negative. The graph $G$ is sign symmetric if for every pair $u$ and $v$ in $V$, all weights of any edges between $u$ and $v$ are either all positive or all negative. An undirected path or cycle in a directed graph $G$ is a non-self-intersecting path or cycle following edges without any regard for their direction. A cycle or path is called positive if the product of the weights encountered along the cycle or path is positive, and negative if that product is negative. A weakly connected component of $G$ is a maximal subgraph all of whose vertices can be connected by undirected paths.

Definition. A directed graph $G$ is called balanced if every undirected cycle is positive.

In the context of social dynamics, this definition is essentially due to Harary [5] and Cartwright and Harary [3] expanding on earlier concepts by Heider [6]. The idea in [3] is that presumably in a state of imbalance "pressures will arise to change it toward a state of balance."

Definition. A directed graph $G$ is said to have two factions if the vertices can be partitioned into (at most) two sets $U_{0}$ and $U_{1}$ such that all edges entirely within $U_{0}$ or within $U_{1}$ have positive weights, while those that connect the two have negative weights.

The main result here is the simple but surprising conclusion that these two definitions are equivalent for undirected graphs. This result, the structure theorem, was proved in the 1950s [3, 5] for undirected graphs. We give a slight generalization here. 
Theorem 1. A directed graph with signed weights $G$ is balanced if and only if it has two factions $U_{0}$ and $U_{1}$.

Proof. Without loss of generality we may assume that $G$ has one (weakly connected) component. By definition, $G$ is balanced if and only if every undirected cycle is positive. But that is true if and only if for all $u$ and $v$ in $V$ any two undirected paths connecting $u$ and $v$ have the same sign. In turn, this is equivalent to partitioning the vertices in $V$ as follows. Start with a vertex $u$ and call its faction $U_{0}$. For any $v \in V$, $v$ is in $U_{\pi}$ where $\pi$ is 0 if the undirected paths connecting $u$ and $v$ are positive, and $\pi$ is 1 if they are negative.

Historical remark. From a graph $G$ with two factions we obtain a bipartite graph $H$ by deleting all its positive edges. One thus sees without much trouble that the above result is in fact equivalent to a much earlier result published by D. König in 1916 [7], namely that a graph is bipartite if and only if it has no odd cycles.

\section{WHEN DOES SOCIAL BALANCE EVOLVE?}

Definition. An $n \times n$ matrix $X$ is called typical if it is a real matrix that satisfies all of the following conditions:

1) $\operatorname{det} X_{0} \neq 0$.

2) $X_{0}$ has a positive real eigenvalue.

3) The largest positive real eigenvalue $\lambda$ of $X_{0}$ is simple.

Let $M_{n}$ be an ensemble of real $n \times n$ matrices whose entries have independent Gaussian distribution with mean zero and variance one. Tao and Vu proved that if $n$ is even, then matrices in $M_{n}$ will have some real eigenvalues with probability tending to 1 as $n$ tends to infinity [12, Corollary 17]. For this reason we assume from now on that $n$ is even and use the characterization typical in the above definition. In addition, most of these eigenvalues will be simple [12, Corollary 18]. Tao and Vu also conjecture, that in fact with overwhelming probability none of the eigenvalues should be repeated.

To facilitate the proof of the next results, we introduce some notation (see Figure 11. Let $X_{0}$ be a typical $n \times n$ matrix with leading (real, simple, positive) eigenvalue $\lambda$. Pick $v$, a unit eigenvector associated with $\lambda$. Denote by $W$ the span of all (generalized) eigenspaces other than $\operatorname{span}\{v\}$. Let $w$ be the vector determined by

$$
w \in W^{\perp} \quad \text { and } \quad(w, v)=1
$$

where $($,$) is the standard inner product in \mathbb{R}^{n}$. It will become clear that in fact $w$ is a left eigenvector of $X_{0}$. Generally $w$ is not a unit vector. The unit vector in the direction of $w$ will be called $u$. If $w$ is a unit vector, then we have $w=v=u$. The hyperplane $W$ separates the unit sphere $S^{n-1}$ into two hemispheres, one of which contains the vector $u$. By construction $u$ and $v$ lie in the same hemisphere which we denote by $N_{u}$. Finally we choose an orthonormal basis $\left\{w_{2}, \ldots, w_{n}\right\}$ for $W$.

Theorem 2. Let $n$ be even and let $X_{0} \in M_{n}$ be a typical $n \times n$ matrix (see Definition 3). Then with probability tending to 1 as $n$ tends to $\infty$, the (matrix-valued) initial value problem

$$
\dot{X}=X^{2} ; \quad X(0)=X_{0}
$$

has a unique solution for $t \in\left[0, \lambda^{-1}\right)$, and near $t=\lambda^{-1}$ the solution diverges as follows ( $v$ and $w$ as defined above):

$$
\lim _{t \nearrow \lambda^{-1}}\left(\lambda^{-1}-t\right) X=v w^{T} .
$$




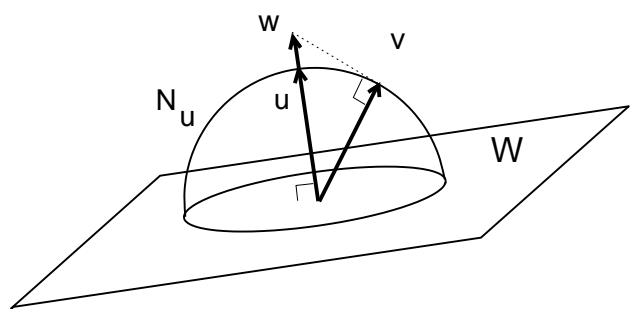

Figure 1. Schematic representation of $W, N_{u}, w, u$, and $v$ in $\mathbb{R}^{n}$.

Proof. Upon substitution of $Z=X^{-1}$, the differential equation given in (1) transforms into

$$
\dot{Z}=-I ; \quad Z(0)=X_{0}^{-1}
$$

(where we used condition 1). The solution is:

$$
Z(t)=X_{0}^{-1}-t I \quad \text { or } \quad X(t)=\left(X_{0}^{-1}-t I\right)^{-1} .
$$

It is unique and exists until it diverges.

Now recall the notation in the paragraph prior to the statement of Theorem 2 . Let $P$ be the matrix whose first column is $v$ and whose $i$ th column is $w_{i}$ for $i>1$ :

$$
P=\left[\begin{array}{llll}
v & w_{2} & \cdots & w_{n}
\end{array}\right] .
$$

By its definition, $P$ is invertible and using conditions 2 and 3 we have

$$
X_{0}=P\left(\begin{array}{l|l}
\lambda & 0^{T} \\
\hline 0 & \tilde{X}_{0}
\end{array}\right) P^{-1}
$$

This uncouples the first component from the rest of the system. By using equation (3), we obtain that, for $t \in\left[0, \lambda^{-1}\right)$,

$$
\begin{aligned}
X(t) & =P\left(\begin{array}{c|c}
\left(\lambda^{-1}-t\right)^{-1} & 0^{T} \\
\hline 0 & \left(\tilde{X}_{0}^{-1}-t I\right)^{-1}
\end{array}\right) P^{-1} \\
& =P\left(\begin{array}{c|c}
\left(\lambda^{-1}-t\right)^{-1} & 0^{T} \\
\hline 0 & \mathbf{0}
\end{array}\right) P^{-1}+P\left(\begin{array}{c|c}
0 & 0^{T} \\
\hline 0 & \left(\tilde{X}_{0}^{-1}-t I\right)^{-1}
\end{array}\right) P^{-1}
\end{aligned}
$$

By hypothesis if $\tilde{X}_{0}$ has real eigenvalues, the largest of these is less than $\lambda$, and so in the interval $\left[0, \lambda^{-1}\right]$ the second term is uniformly bounded by a constant.

Finally, $P^{-1} P=1$ implies that the first row $r$ of $P^{-1}$ satisfies

$$
(r, v)=1 \quad \text { and } \quad \text { for all } \mathrm{i}>1,\left(\mathrm{r}, \mathrm{w}_{\mathrm{i}}\right)=0 .
$$

Therefore, the first row of $P^{-1}$ equals $w^{T}$. Equation (5) now gives

$$
X(t)=\left(\lambda^{-1}-t\right)^{-1} v w^{T}+\mathcal{O}(1) .
$$

Multiplying both sides by $\left(\lambda^{-1}-t\right)$ implies the theorem. 
Remark. By transposing equation (4), we see that in fact $w$ is a eigenvector of $X_{0}^{T}$ associated with $\lambda$. Equivalently, it is a left eigenvector of $X_{0}$.

Remark. The blow-up at finite time may be avoided by dividing the vector field in equation (1) by $1+|X|$, where $|X|$ is any suitable matrix norm. The vector fields defined by $X^{2}$ and $\frac{X^{2}}{1+|X|}$ have the same direction, and so the phase portrait of the two systems is the same [4, Section 1.5]. The effect of the scalar factor is simply to slow down the flow. Doing this explicitly leads to very complicated equations without adding insight. We follow the literature on the subject and do not pursue this.

Corollary 1. If $X_{0}$ satisfies the hypotheses of Theorem 2 then

$$
\lim _{t \nearrow \lambda^{-1}}\left(\lambda^{-1}-t\right) X=v v^{T}
$$

if and only if the left and right eigenvectors associated to $\lambda$ of $X_{0}$ are the same.

This easily implies that for $t$ close to $\lambda^{-1}$ and up to permutation of the coordinates, $X(t)$ has the block form

$$
\operatorname{sgn} X(t)=\left(\begin{array}{l|l}
+ & - \\
\hline- & +
\end{array}\right)
$$

Using the terminology of the previous section, this means that the individuals have split into two factions (one possibly being empty). These factions are cohesive in the sense that individuals within a faction like each other. The cases discussed in the literature are where $X_{0}$ is symmetric $[\mathbf{8}]$ or normal [14]. In these cases all eigenspaces are orthogonal, and so we easily recover that $v \in W^{\perp}$. One does need to prove that in the more restricted setting of symmetric or normal matrices, the typical case still has overwhelming probability. This is done in the literature cited.

We can improve Corollary 1 somewhat by noticing the following.

Corollary 2. If $X_{0}$ satisfies the hypotheses of Theorem 2 then for t close to $\lambda^{-1}, X(t)$ has two factions if and only if the left and right eigenvectors associated with $\lambda$ fall in the same orthant, i.e., their components have the same signs.

4. WHAT IF SOCIAL BALANCE DOES NOT EVOLVE? Even the early sociological literature admits that the assumption that the matrix $X$ is symmetric, or even sign-symmetric, is unrealistic [6]. It is therefore natural to inquire what happens in this model if we drop that assumption.

Theorem 2 implies that for $t$ close to $\lambda^{-1}$ the matrix $X(t)$ has the sign pattern of $v w^{T}$. Permute the components so that the first $K$ components of $v$ are positive and the others negative. After that, permute the first $K$ components so that among them, the positive components of $w$ and the negative ones are grouped together, and so forth. It follows that for $t$ close to $\lambda^{-1}, X$ has the following sign pattern:

$$
\operatorname{sgn} X(t)=\left(\begin{array}{cc|cc}
+ & - & + & - \\
+ & - & + & - \\
\hline- & + & - & + \\
- & + & - & +
\end{array}\right) .
$$

Notice that this matrix is not sign symmetric and thus the system is not balanced. The evolution towards this pattern is illustrated in Figure 2 . 

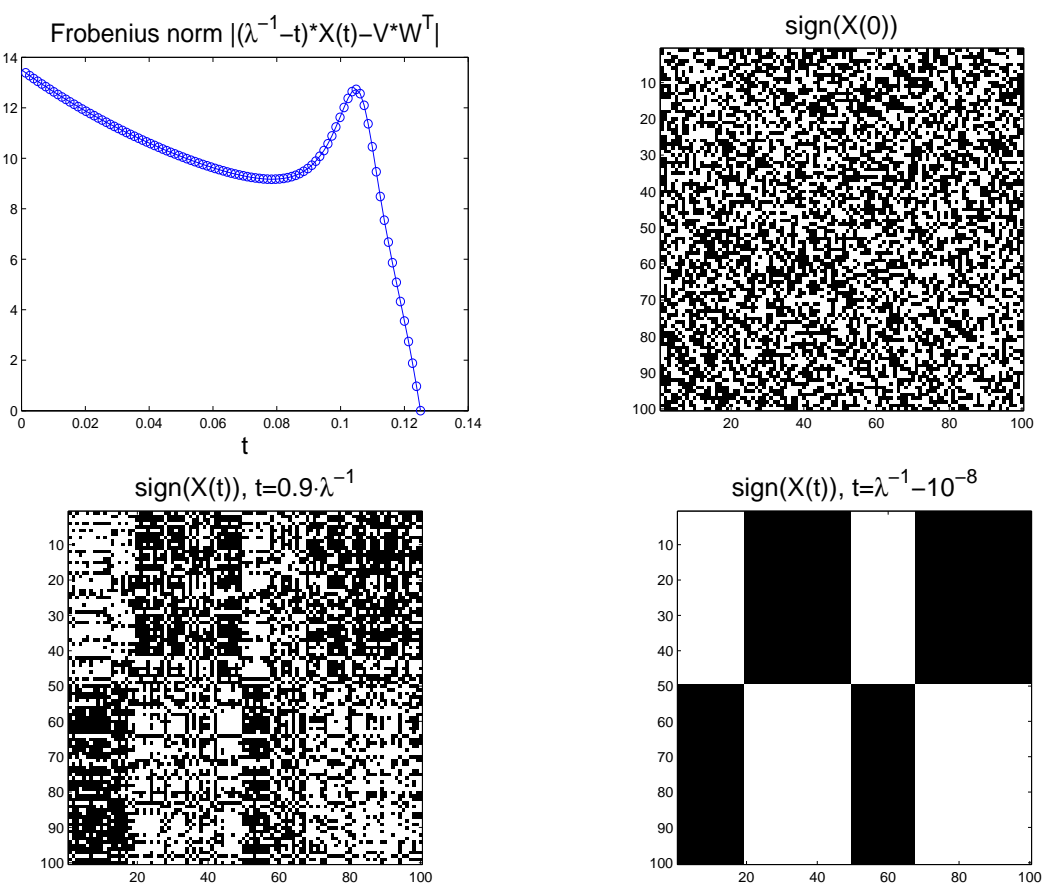

Figure 2. The upper left diagram illustrates Theorem 2 for a 100 by 100 system $(\lambda \approx 8.01)$. The Frobenius norm is the root of the sum of the squares of the matrix entries. The convergence to the sign pattern of Equation (6) is illustrated in the next three figures. After the appropriate permutation of the components, we show the signs of the initial configuration $X(t)$ when $t=0, t=0.9 \cdot \lambda^{-1}$, and $t=\lambda^{-1}-10^{-8}$, respectively. (White signifies a positive entry, while black signifies negative.)

Thus there still are two balanced or cohesive factions (that is, factions within each of which individuals like each other). But now there are also two dispersive factions that are internally divided in the sense that individuals within either one of these factions dislike each other (they even dislike themselves), signifying diagonal blocks of negative entries (entries $(2,2)$ and $(3,3)$ in equation (6) $)$. Note also that the dispersive factions have symmetric feelings about each other (for example, entries $(2,3)$ and $(3,2)$ are both positive), but the relations between a cohesive and a dispersive faction are anti-symmetric (for example, entries $(2,4)$ and $(4,2)$ have opposite signs).

Let us name the factions 1, 2, 3, and 4, in the order of their appearance along the diagonal of equation (6). Adhering to the interpretation that $x_{i j}$ corresponds to the opinion of $i$ towards $j$, one might speculate that the fact that faction 2 is disliked by faction 1 while they still have positive feelings for faction 1, perhaps caused them to feel ill at ease and have a negative opinion about themselves. At the same time, they cannot join faction 4 because they dislike them. Mutual sympathy exists, however, between factions 3 and 4 . Whether or not this is reasonable from a sociological point of view, is unclear to us.

This brings us to the interesting question of which alliances of two factions out of four would likely give a majority? By symmetry, eigenvectors $v$ and $w$ are equally likely as $-v$ and $-w$. This would swap factions 1 and 4 on the one hand, and factions 2 and 3 on the other. Thus the cohesive factions 1 and 4 have the same expected size, and the same holds for the dispersive factions. The alliance of factions 1 and 2 has the same size as the number of "+" signs in the vector $v$. Again by symmetry, the 
expectation is exactly one half times $n$. Therefore they will form a majority half the time. Therefore the same is true for any combination of a dispersive and a cohesive faction. However, if the larger of the two cohesive factions combines with any of the dispersive factions, then the expectation is that it will have a majority.

The hard part is to decide what will happen if the dispersive factions (factions 3 and 4) join forces, as was suggested above. In the remainder of the section, we argue that if the two dispersive factions get together, they are still expected to be a minority.

We use the notation of Section 3 (see also Figure1).

Theorem 3. Let $X_{0}$ satisfy the hypotheses of Theorem 2 Assume furthermore that $v$ is uniformly distributed in $N_{u}$. Then the expected number of " + " signs on the diagonal of the matrix in equation (6) lies in the interval $\left(\frac{n}{2}, \frac{n}{2}\left(1+\sqrt{\frac{2}{\pi n}}\right)\right)$.

Define $f_{u}: N_{u} \rightarrow \mathbb{Z}$ by

$$
f_{u}(v)=\sum_{i=1}^{n} \operatorname{sgn} v_{i} \operatorname{sgn} w_{i}=\sum_{i=1}^{n} \operatorname{sgn} v_{i} \operatorname{sgn} u_{i}=n-2 k,
$$

where $k$ is the number of sign disagreements between the components of the vectors $v$ and $u$. Recall that by definition $v$ lies in $N_{u}$ (see Figure 1). If $v$ is uniformly distributed in $N_{u}$, then the expected value of $n-2 k$ equals

$$
I_{u}^{+}=\frac{1}{\operatorname{vol} N_{u}} \int_{N_{u}} f_{u}(v) d S^{n-1},
$$

where $d S^{n-1}$ is the density of the Lebesgue measure on $S^{n-1}$. In the following, $I_{u}^{-}$ denotes the integral of $f_{u}(v)$ over the complement of $N_{u}$ in $S^{n-1}$. Theorem 3 counts the expected number $n-k$ of sign agreements and is thus a direct consequence of the following proposition.

Proposition 1. Let $n$ be even. Given $u \in S^{n-1}$, then

$$
0<I_{u}^{+}<\sqrt{\frac{2 n}{\pi}} .
$$

Proof. The first inequality follows from the following two claims:

$$
I_{u}^{+}+I_{u}^{-}=0 \quad \text { and } \quad I_{u}^{+}>I_{u}^{-} .
$$

The first of these claims follows from the observation that $I_{u}^{+}+I_{u}^{-}$is the integral over all of $S^{n-1}$. Since $f_{u}(-v)=-f_{u}(v)$, this must yield zero. With probability one, all $u_{i}$ are nonzero. Therefore, for every $i$, any geodesic arc $\gamma(t)$ connecting $-u$ to $u$ must cross the equator $u_{i}=0$ exactly once. Thus on such an arc $\left.\operatorname{sign} u_{i} \operatorname{sign} v_{i}\right|_{\gamma(t)}$ is nondecreasing, and increases from -1 to 1 . The second claim follows.

To get the other inequality, we first partition $S^{n-1}$ into $2^{n}$ orthants $O_{\sigma}$. For every $\sigma \in\{-1,+1\}^{n}$,

$$
O_{\sigma}=\left\{v \in S^{n-1} \mid\left(\operatorname{sgn} u_{1} \operatorname{sgn} v_{1}, \operatorname{sgn} u_{2} \operatorname{sgn} v_{2}, \ldots, \operatorname{sgn} u_{n} \operatorname{sgn} v_{n}\right)=\sigma\right\} .
$$

Now let the set $Q$ be the quotient of the sequences $\{-1,+1\}^{n}$ and multiplication by -1 . Because $n$ is even, $Q$ can be parametrized by those binary sequences $s$ that have positive average plus half of the ones that have zero average. Define

$$
Z_{s}=\left(O_{s} \cup O_{-s}\right) \cap N_{u} .
$$


The sets $Z_{s}$ partition $N_{u}$ into $2^{n-1}$ sets of equal measure (up to measure zero). For all $v$ in the set $Z_{s}, f_{u}(v)$ equals either $\sum_{i=1}^{n} s_{i}$ or $\sum_{i=1}^{n}\left(-s_{i}\right)$. Because $\sum_{i=1}^{n} s_{i}$ is equal to $n-2 k$ where $k$ is the number of sign differences,

$$
I_{u}^{+} \leq 2^{1-n} \sum_{s \in Q}\left(\sum_{i=1}^{n} s_{i}\right)=2^{1-n} \sum_{s \in Q}(n-2 k)
$$

in $Z_{s}$. Because $k=\frac{n}{2}$ ( $n$ is even) contributes 0 to the above sum, we can replace the sum over $Q$ by the sum over $Q^{\prime}$, the set of all sequences in $\{0,1\}^{n}$ whose average is at least zero. There are $\left(\begin{array}{l}n \\ k\end{array}\right)$ distinct sequences $s$ in $Q^{\prime}$ that have $k$ sign differences, so

$$
\begin{aligned}
2^{1-n} \sum_{s \in Q^{\prime}}(n-2 k) & =2^{1-n} \sum_{k=0}^{\frac{n}{2}}\left(\begin{array}{l}
n \\
k
\end{array}\right)[(n-k)-k] \\
& =2^{1-n} \sum_{k=0}^{\frac{n}{2}} n\left[\left(\begin{array}{c}
n-1 \\
k
\end{array}\right)-\left(\begin{array}{c}
n-1 \\
k-1
\end{array}\right)\right]=2^{-n} n\left(\begin{array}{c}
n \\
\frac{n}{2}
\end{array}\right)
\end{aligned}
$$

by telescoping (with the convention that $\left(\begin{array}{c}n-1 \\ -1\end{array}\right):=0$ ). Now use Stirling's formula in the following form [9, Section 3.6]:

$$
n !=\sqrt{2 \pi n}\left(\frac{n}{e}\right)^{n}\left(1+\frac{1}{12 n}+\frac{1}{288 n^{2}}+\cdots\right) .
$$

Proposition 1 follows after some straightforward algebra.

The prediction of Theorem 3 depends on the assumption that $v$ is uniformly distributed in $N_{u}$. If the distribution $d \rho$ of $v$ is more biased towards the vicinity of the pole $u$ in $N_{u}$, then the majority of the united cohesive factions will be more pronounced. On the contrary, if the distribution is more biased towards the equator, then that majority will be narrower than described here.

ACKNOWLEDGMENT. I am grateful to Patrick de Leenheer for his insightful comments and indebted to Dacian Daescu for providing the software used to make figure displaying the simulation.

\section{REFERENCES}

1. Arnold, L. (1971). On Wigner's semicircle law for the eigenvalues of random matrices, Z. Wahrscheinlichkeitstheorie Verw. Geb. 19: 191-198.

2. Bernoulli, J. (1695). Explicationes, Annotationes \& Additiones ad ea, quae in Actis sup. de Curva Elastica, Isochrona Paracentrica, \& Velaria, hinc inde memorata, \& paratim controversa legundur; ubi de Linea mediarum directionum, alliisque novis, Acta Eruditorum: 537-553

3. Cartwright, D., Harary, F.(1956). Structural balance: a generalization of Heider's theory, Psychological Review 63(5): 277-293.

4. Chicone, C. (2006). Ordinary Differential Equations, 2nd Ed. New York: Springer.

5. Harary, F. (1953). On the Notion of Balance of a Signed Graph, Michigan Math. J. 2(2): 143-146.

6. Heider, F. (1946). Attitudes and Cognitive Organization, J Psychol 21: 107-112.

7. König, D. (1916). Über Graphen und Ihre Anwendung auf Determinantentheorie und Mengenlehre, Mathematische Annalen 77: 453-465.

8. Marvel, S., Kleinberg, J., Kleinberg, R., Strogatz S. (2011). Continuous-time model of structural balance, Proceedings of the National Academy of Sciences 108(5): 1771-1776.

9. Mathews, J., Walker, J. R. (1970). Mathematical Methods of Physics, 2nd ed. New York: W. A. Benjamin.

10. Rapoport, A. (1963). Mathematical models of social interaction. In: Galanter, R. A., Lace, R. R., Bush, E., eds. Handbook of Mathematical Sociology, Vol 2. New York, NY: Wiley \& Sons, pp. 493-580. 
11. Reid, W. T. (1946) A matrix differential equation of Riccati type, American Journal of Mathematics 68(2): 237-246.

12. Tao, T., Vu, V. (2015). Random matrices: Universality of local spectral statistics of non-Hermitian Matrices, Annals Prob 43(2): 782-874.

13. Teschl, G. (2012). Ordinary Differential Equations and Dynamical Systems, Graduate Studies in Mathematics, 140, Providence: American Mathematical Society.

14. Traag, V. A., Van Dooren, P., De Leenheer, P. (2013). Dynamical models explaining social balance and evolution of cooperation, PLOS ONE 8(4): e60063.

J. J. P. VEERMAN received his Ph.D. from Cornell University. He has held visiting positions in the U.S. (Rockefeller University, Georgia Tech, Penn State), as well as in Spain, Brazil, Italy, and Greece. He is currently at Portland State University in Oregon, USA, where he is Professor of Mathematics.

Maseeh Department of Mathematics and Statistics, Portland State University, Portland, OR 97201.

veerman@pdx.edu 